\section{A program for simultaneously transforming two variables to maximize linear regression, normality, and homoscedasticity}

JEFFREY LEE RASMUSSEN

and

\author{
WILLIAM P. DUNLAP \\ Tulane University, New Orleans, Louisiana
}

When a researcher is faced with two variables, $X$ and $\mathrm{Y}$, that show a nonlinear relationship, the use of these variables in a regression analysis may be hampered unless the variables can be transformed in such a way as to obtain a linear relationship. Furthermore, if transformations can be found to produce linearity, they may aid in understanding the underlying functional relationship. Dunlap and Rasmussen (1982) used the Box and Cox (1964) family of transformations in a program that first transforms $X$ to obtain the best linear prediction of $Y$ and then transforms $Y$ to get the best prediction of untransformed $X$. The program described in this paper deals with the more general and more difficult problem of finding simultaneous transformations of both $\mathrm{X}$ and $Y$ to optimize the linear relation between the two.

The Box and Cox (1964) family of transformations depends upon the single parameter lambda $(\lambda)$, such that $\mathrm{X}^{*}=\left(\mathrm{X}^{\lambda}-1\right) / \lambda$, where $\mathrm{X}$ represents the raw data and $\mathrm{X}^{*}$ the transformed score. When $\lambda=-1$, this function produces the reciprocal transformation, when $\lambda=0$, it produces the logarithmic transformation, when $\lambda=.5$, it produces the square root, and when $\lambda=1$, the data are unchanged. The goal of the present program is to find a $\lambda_{x}$ for $X$ and a $\lambda_{y}$ for $Y$ such that the simple correlation between $\mathrm{X}^{*}$ and $\mathrm{Y}^{*}$ is maximized. Although it would seem to be a simple matter to first transform $X$, then $Y$, and then $X$ again, etc., until the process converges, Draper and Hunter (1969) reported that such a procedure does not converge. The reason is that the surface representing $r$ as a joint function of $\lambda_{y}$ and $\lambda_{\mathbf{x}}$ is often saddle shaped, such that the actual maxima are pathological cases in which normality and/or homogeneity of variance about the regression line are badly violated.

The choice of a best set of $\lambda_{y}$ and $\lambda_{x}$ values can be made by satisfying four criteria. First, those values that produce greater linearity (larger $r$ ) should be chosen over those that produce nonlinearity. Second, those $\lambda$ values that tend to produce homogeneity of variance within the

The first author's mailing address is: Department of Psychology, University of Arizona, Tucson, Arizona 85721; the second author's mailing address is: Department of Psychology, Tulane University, New Orleans, Louisiana 70118.
$\mathrm{Y}$ and $\mathrm{X}$ arrays should be chosen. A practical consequence of homoscedasticity within arrays is that the error of prediction of $Y$ values is consistent across values of $X$ and vice versa. A third criterion for choosing transformation values is to maximize normality of the projections of the $X Y$ points on the regression line. A practical problem is that spuriously high correlations may result due to outliers if the data do not fall in at least an approximately symmetrical distribution. Statistical estimation and prediction, such as the setting of confidence intervals and the testing of hypotheses, depend upon the extent to which the underlying assumptions of normality and homoscedasticity within arrays are met (Binder, 1959). Nonnormality, for example, can lead to severe departures of the actual from the theoretical sampling distribution of $\mathrm{r}$ with highly correlated variables (Norris \& Hjelm, 1961). The fourth criterion is to choose those $\lambda$ values that lead to the most parsimonious, logically coherent relationship between the two variables (see Draper \& Hunter, 1969). This last criterion, of course, presupposes that the researcher has some theoretical reason to pick one set of values over another.

The present program supplies the user with information on the three criteria of linearity, homoscedasticity, and normality for the various values of $\lambda_{y}$ and $\lambda_{x}$. The measure of linearity is Pearson's product-moment correlation coefficient. Since Pearson's $r$ measures the extent to which a straight line fits the data, larger $r$ values correspond to greater linearity of regression.

Homogeneity of Variance. The index of homogeneity of variance within arrays is a modification of Levene's (1960) statistic. For each XY transformation, a correlation coefficient is calculated between the data points' projection along the first eigenvector and the data points' absolute deviation from the first eigenvector. If the data are in standardized form, the first eigenvector (a 45deg line passing through the origin) is the best-fitting nondirectional linear-regression line. The deviation of a data point is equal to the length of a perpendicular line from the first eigenvector to the data point. The deviation of a data point $(X, Y)$ from the nondirectional regression line is $(\mathrm{Y}-\mathrm{X}) / 2^{1 / 2}$. To the extent that the absolute deviations of points from the regression line increase or decrease with distance along the regression line, the absolute value of this correlation increases or decreases; therefore this correlation provides an index of heteroscedasticity.

Normality Along the Regression Line. To measure normality of the projections of the data points along the nondirectional regression line, the following procedure is used. First, the projections are converted to standard form (mean $=0, s=1$ ) and ordered from least to greatest. Next, a set of expected ordered normal scores is generated using a subroutine by Dunlap and Brown (1983). Last, the average absolute deviation of the or- 
dered standardized projections from the ordered expected normal deviates is computed. This index is sensitive to deviations from normality in terms of skew or kurtosis. Low values indicate approximate normality; higher values indicate nonnormality.

Program Execution. The program works as follows. First, the user inputs the data. Next, the data are searched for negative or zero values. If any are found, the absolute value of the minimum datum plus one is added to each score before the data are transformed, because $\lambda$ is defined only for nonnegative values. A scatterplot of the raw data is displayed on the CRT screen. The user is then informed of the default range of $\lambda$ values from -3 to 3 and is given the opportunity to change the range. Sixteen equally spaced values of $\lambda_{y}$ in the specified range are used in conjunction with 31 values of $\lambda_{x}$; thus, a total of 496 transformation pairs are carried out. For each transformation, indices for correlation, homoscedasticity, and normality are calculated. The program rescales the values of these indices from 0 to 9 by subtracting the minimum value and dividing by the range for each value. These rescaled values are arranged in separate contour maps for each index and are written to an output file, along with a key that indicates corresponding actual values (see Figure 1). A composite contour map of the maximum $r$ values with the minimum homoscedasticity and normality indices is displayed on the screen (lower right panel in Figure 1).

The contour maps are $16 \times 31$ matrices that indicate the relative value of a statistic for each of the $Y$ and $X$ lambda values. The maps tend to create a pattern of values that identify those transformations that serve to maximize $r$, homogeneity, and normality. The map of correlations, for example, shows a typical saddle-shaped pattern indicating that many $\mathrm{XY}$ transformations serve to maximize r. Inspection of the contour maps for homoscedasticity and normality, however, indicates that many of these $\mathrm{XY}$ transformations create pathological cases in which normality and homoscedasticity are seriously compromised. To aid the researcher in picking the best transformation values, the composite map is created from the separate maps. For each $Y$ and $X$ lambda value, there are six possible symbols that may appear in the composite map. A " " " indicates that the particular XY $\lambda$ transformation does not meet any of the three criteria for a good transformation. An " $R$ " indicates that the $\mathrm{XY} \lambda$ values produce the highest $10 \%$ of linearity of regression between $\mathrm{Y}$ and $\mathrm{X}$. An " $\mathrm{H}$ " indicates that the $\mathrm{XY} \lambda$ values produce the lowest $10 \%$ of heterogeneity of variance within arrays. An "N" indicates that the transformation produces the lowest $10 \%$ of nonlinearity along the regression line. A " 2 " indicates that the transformation meets two of the criteria (i.e., maximum linearity and minimum heterogeneity; maximum linearity and minimum nonnormality; or minimum he terogeneity and minimum nonnormality). A " 3 " indicates that the transformation meets all three criteria. Figure 1 shows that $\lambda_{\mathrm{y}}=.2, \lambda_{\mathrm{x}}=.4$ serves to simultaneously maximize $\mathrm{r}$ and minimize heteroscedasticity and nonnormality. In

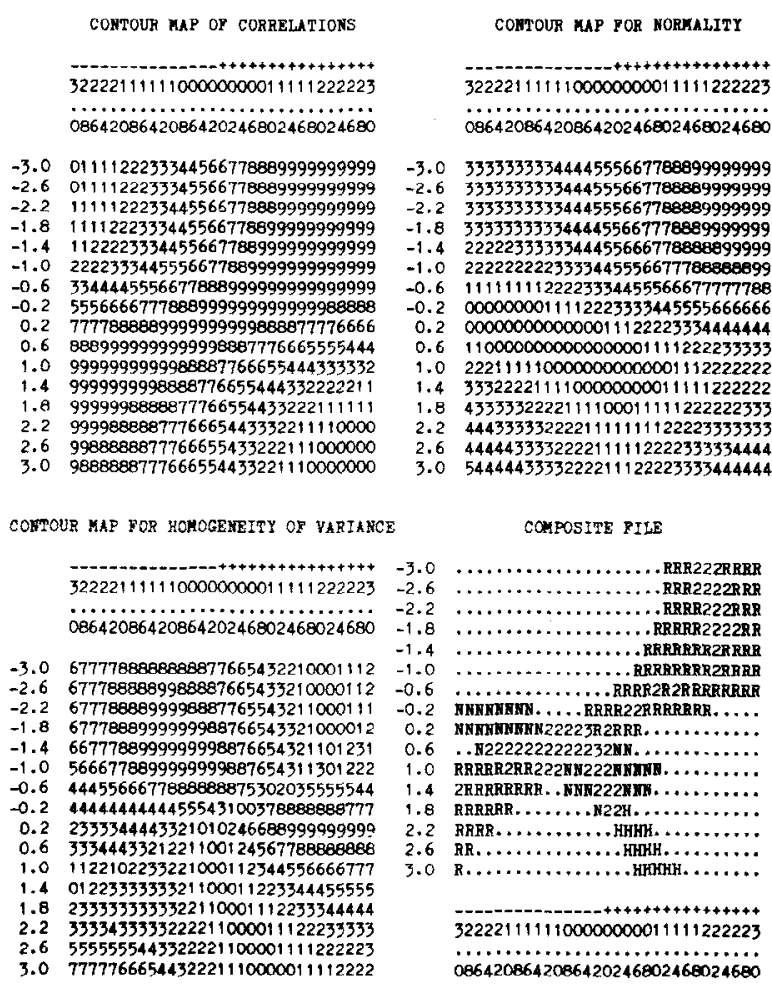

Figure 1. Contour maps for correlation, normality, and homogeneity of variance; and a composite map (lower right panel) for maximum correlation (R), normality $(\mathrm{N})$, and homoscedasticity (H).

the example, the program produces a contour map that shows values of " $N$," " $H$," and " $R$ " converging upon a central area of " $2 \mathrm{~s}$ " surrounding two values of " 3 ." In other instances, the contour map may indicate that the best transformation values are off the map or that the user should focus on a smaller area of the map. In either case, the program allows the user to pick different minimum and maximum $\lambda$ values, and it produces another contour map with values within the new range. Finally, the user is asked to select a best pair of $\lambda_{y}$ and $\lambda_{x}$ values. A scatterplot of the data points transformed by these $\lambda_{y}$ and $\lambda_{x}$ values is shown, and a plot of the deviations of the points from the first eigenvector is displayed.

Requirements. The program is written in singleprecision FORTRAN IV and runs on a DEC $2060 \mathrm{com}$ puter. Since the program is interactive, most of the input and output is to the computer terminal, although data may be read from a file. There are a limited number of nonstandard FORTRAN statements used to make the program run conveniently on the DEC system. Users with other systems will probably need to modify assignments of logical units, READ or WRITE statements with an "*" in the usual place of a format statement number (this causes free format on the DEC), and input format statements with the final symbol " $\$$," which causes the cursor to remain at the end of a line on the DEC system. The FORTRAN source program requires 
about $17 \mathrm{~KB}$ of memory. The run time required for the program depends upon the number of cases processed. For example, running the program on the DEC 2060 requires approximately $3 \mathrm{CPU}$ sec for 10 cases, $22 \mathrm{CPU}$ sec for 50 cases, $48 \mathrm{CPU}$ sec for 100 cases, and $130 \mathrm{CPU}$ sec for 200 cases.

Program Availability. A listing of the program is available at no charge from Jeffrey Lee Rasmussen, Department of Psychology, University of Arizona, Tucson, Arizona 85721.

\section{REFERENCES}

Binder, A. Considerations of the place of assumptions in correlational analysis. American Psychologist, 1959, 14, 504-510.

Box, G. E. P., \& Cox, D. R. An analysis of transformations.
Journal of the Royal Statistical Society (Series B), 1964, 26, 211-243.

Draper, N. R., \& Hunter, W. G. Transformations: Some examples revisited. Technometrics, 1969, 11, 23-40.

DunlaP, W. P., \& Brown, S. G. Fortran IV functions to compute expected normal scores. Behavior Research Methods \& Instrumentation, 1983, 15, 395-397.

Dunlap, W. P., \& Rasmussen, J. L. A program to determine data transformations maximizing linear regression. Behavior Research Methods \& Instrumentation, 1982, 14, 357-358.

LEVENE, H. Robust tests for equality of variances. In I. Olkins (Ed.), Contributions to probability and statistics. Stanford, Calif: Stanford University Press, 1960.

Norris, R. C., \& HJELM, H. F. Non-normality and product moment correlation. Journal of Experimental Education, 1961, 29, 261-270.

(Manuscript accepted for publication July 7, 1983.) 